\title{
Factors associated with habitual sleep duration in US adults with hypertension: a cross- sectional study of the 2015-2018 National Health and Nutrition Examination Survey
}

\author{
Everlyne G. Ogugu ${ }^{1 *}$, Sheryl L. Catz ${ }^{1}$, Janice F. Bell ${ }^{1}$, Christiana Drake ${ }^{2}$, Julie T. Bidwell ${ }^{1}$ and James E. Gangwisch ${ }^{3}$
}

\begin{abstract}
Background: The relationship between inadequate sleep duration and hypertension risk has been established in the general population, but there is a gap in the literature on predictors of habitual sleep duration in adults with hypertension. This study examined factors associated with habitual sleep duration among adults with hypertension in the United States (US).

Methods: Data of 5660 adults with hypertension were obtained by combining the 2015-2018 cycles of the National Health and Nutrition Examination Survey (NHANES). Survey weighted multinomial logistic regression models were fit to examine factors associated with short ( $<7 \mathrm{~h}$ ) and long $(>9 \mathrm{~h})$ sleep duration with adequate sleep duration $(7-9 \mathrm{~h})$ as the reference.

Results: The prevalence of self-reported adequate sleep duration was $65.7 \%$, while short sleep duration was $23.6 \%$, and long sleep duration $10.7 \%$. Short sleep duration (compared to adequate sleep duration) was positively associated with history of seeking help for sleeping difficulties (relative risk ratio [RRR], 1.25; 95\% confidence interval [Cl], 1.02-1.53), Non-Hispanic Black race/ethnicity (RRR, 2.08; $95 \% \mathrm{Cl}, 1.61-2.67$ ), working $\geq 45 \mathrm{~h} /$ week (RRR, 1.81; $95 \%$ $\mathrm{Cl}, 1.32-2.48$ ), and negatively associated with older age $\geq 65$ years (RRR, $0.63 ; 95 \% \mathrm{Cl}, 0.45-0.91$ ) and female gender (RRR, 0.70; 95\% Cl, 0.56-0.88). Long sleep duration was positively associated with female gender (RRR, 1.24; 95\% Cl, 1.001-1.54), chronic kidney disease (RRR, 1.48; $95 \% \mathrm{Cl}, 1.14-1.92)$, moderate depressive symptoms (RRR, 1.62; 95\% Cl, 1.08-2.44), moderately severe to severe depressive symptoms (RRR, 1.89; 95\% Cl, 1.05-3.43), being in retirement (RRR, $3.46 ; 95 \% \mathrm{Cl}, 2.18-5.49$ ), and not working due to health reasons (RRR, 4.87; 95\% Cl, 2.89-8.22) or other reasons (RRR, $3.29 ; 95 \% \mathrm{Cl}, 1.84-5.88)$.

Conclusion: This population-based study identified factors independently associated with habitual sleep duration in adults with hypertension. These included help-seeking for sleeping difficulty, gender, age, chronic kidney disease, depressive symptoms, race/ethnicity, and employment status. These findings can help in the development of tailored approaches for promoting adequate sleep duration in adults with hypertension.
\end{abstract}

Keywords: Sleep duration, Self-reported sleep, Blood pressure, Hypertension, NHANES

*Correspondence: egogugu@ucdavis.edu

1 Betty Irene Moore School of Nursing, University of California, Davis 2570

48th Street, Sacramento, CA 95817, USA

Full list of author information is available at the end of the article

\section{Introduction}

Sleep is crucial for general wellbeing and physiologic function, including blood pressure (BP) regulation [1]. The American Academy of Sleep Medicine and Sleep 
Research Society (AASM/SRS) and the National Sleep Foundation (NSF) recommend that individuals aged $\geq 18$ years sleep for a minimum of $7 \mathrm{~h}$ a day to stay healthy $[1,2]$. However, the proportion of United States (US) adults who habitually sleep less than the recommended hours has increased over the years. For example, the prevalence of habitual short sleep duration $(<7 \mathrm{~h} /$ day) among working US adults rose from $30.9 \%$ in 2010 to $35.6 \%$ in 2018 [3]. The burden of hypertension also remains a public health challenge in the US. Based on the 2017 American College of Cardiology and American Heart Association (ACC/AHA) guidelines, $47.3 \%$ of US adults have hypertension, and only $26.1 \%$ of those with hypertension have it under control [4].

The 2017 ACC/AHA guidelines recommend weight reduction, adopting a healthy diet, sodium reduction, regular physical activity, and reducing alcohol intake as the primary evidence-based lifestyle interventions for addressing modifiable risk factors of hypertension [5]. However, growing evidence indicates that short sleep duration is also a significant risk factor for hypertension [6-9]. In addition, other studies have reported associations between short sleep duration and factors that can negatively impact hypertension prevention and control, such as obesity $[10,11]$, unhealthy diet $[12,13]$, and increased alcohol intake [14]. These findings on the relationship of sleep duration with hypertension and other health-related behaviors indicate the potential role of habitual sleep duration in preventing and controlling hypertension.

Although studies have identified predictors of habitual sleep duration across different US populations $[15,16]$, none has focused specifically on individuals with hypertension. Given the evidence from many studies of associations between sleep duration and hypertension and its risk factors, it is crucial to examine habitual sleep duration in individuals with hypertension. A better understanding of the determinants of habitual sleep duration in those with hypertension will contribute to more targeted clinical and public health interventions aimed at groups most prone to having inadequate sleep duration. This study's objective was to identify sociodemographic and health factors independently associated with selfreported habitual sleep duration among US adults with hypertension.

\section{Methods}

\section{Study population}

This cross-sectional study used data collected from US adults aged 18 and above in the 2015-2016 and 2017-2018 waves of the National Health and Nutrition Examination Survey (NHANES). The NHANES is a cross-sectional survey whose aim is to estimate the health and nutritional status of civilian, noninstitutionalized US adults and children of all ages. The survey uses complex stratified multistage sampling to get a nationally representative sample. The data is collected through interviews done in a study participant's home and physical examinations completed at a designated mobile examination center (MEC) [17].

The 2015-2016 and 2017-2018 NHANES cycles had 19,225 study participants [18]. Of these, there were 11,848 adults aged 18 and older. The primary inclusion criteria for the present study was the presence of hypertension in an adult aged 18 years and above. In the present study, average systolic BP and diastolic BP were calculated from all the recorded BP measurements taken at the MEC. The NHANES participants had three consecutive BP readings taken after 5 min of rest [19]. Based on the 2017 ACC/AHA guidelines [5] and past studies [20,21], hypertension is defined as the current use of antihypertensive medication in those with a history of hypertension $(n=3215)$, or an average systolic $\mathrm{BP} \geq 130 \mathrm{mmHg}(n=3744)$ or diastolic BP $\geq 80 \mathrm{mmHg}$ $(n=2323)$. The number of adults who met the criteria of hypertension was 5851 .

Out of the remaining 5851, 189 participants were excluded for the following reasons: 7 were pregnant (selfreported pregnant status or a positive urine pregnancy test), 50 were missing data on sleep duration, and 139 only participated in household interviews. Thus, the final study sample of adults with hypertension after exclusions was 5660 .

\section{Outcome measures}

The self-reported habitual sleep duration assessment was based on the amount of sleep usually obtained during weekdays or workdays during the main sleep period (day or night). In the 2015-2018 NHANES cycles, the sleep duration was calculated from responses to two survey questions, "What time do you usually fall asleep on weekdays or workdays?" and "What time do you usually wake up on weekdays or workdays?". In the present study, we categorized habitual sleep duration into short $(<7 \mathrm{~h})$, adequate $(7-9 \mathrm{~h})$, and long $(>9 \mathrm{~h})$ based on guidelines by AASM/SRS and NSF $[1,2]$ and past studies $[14,15]$.

\section{Covariate measures}

Previous studies $[9,13,14,16]$ and Grandner's Socioecological Model of Sleep and Health [22] were used as a guide in identifying covariates to include in the study. Grandner's model outlines factors at the "individual, social and societal levels" related to sleep [22]. The current study focused on factors at the individual (e.g., health behaviors and other health-related characteristics) and social level (e.g., socioeconomic status, employment 
status, and race/ethnicity) of Grandner's model to identify potential determinants of habitual sleep duration, all measured at the individual level of analysis [22].

The "individual-level" covariates included in this study were gender (male, female), age, body mass index, helpseeking for sleeping difficulty, depressive symptoms, chronic health conditions, cigarette smoking, alcohol intake, and physical activity. Age was categorized as 18-44years (young adults), 45-64years (middle-aged adults), and $\geq 65$ years (older adults). Body mass index (BMI) was categorized as $<25$ (no overweight or obesity), 25 to $<30$ (overweight), 30 to $<35$ (obesity class I), 35 to $<40$ (obesity class II), and $\geq 40 \mathrm{~kg} / \mathrm{m}^{2}$ (obesity class III), based on the World Health Organization (WHO) guidelines [23]. Help-seeking for sleeping difficulty was categorized as yes or no based on the response to the question "Have you ever told a doctor or other health professional that you have trouble sleeping?" Depressive symptoms were screened using the 9-item Patient Health Questionnaire (PHQ-9, possible range of 0-27), categorized based on the established criteria of minimal or none (0-4), mild (5-9), moderate (10-14), and moderately severe to severe $(\geq 15)$ depressive symptoms [24].

Chronic health conditions were assessed based on either self-reported history (being informed by a doctor or other health professional of having the specific health condition) or laboratory test findings done at the mobile examination centers. Stroke, heart disease (coronary heart disease, angina, heart attack, or congestive heart failure), arthritis (including gout), and chronic obstructive pulmonary disease (including chronic bronchitis and emphysema) were self-reported. Those who answered in the affirmative to both "Has a doctor or health professional ever told you that you have asthma?" and "Do you still have asthma?" were classified as having asthma. Diabetes was defined as either a self-reported history of diabetes or blood glycohemoglobin $\geq 6.5 \%$. Chronic kidney disease (CKD) was defined as self-reported history of undergoing dialysis in the previous 12 months or an estimated glomerular filtration rate (eGFR) less than $60 \mathrm{~mL} /$ $\min / 1.73 \mathrm{~m}^{2}$ computed using the Chronic Kidney Disease Epidemiology Collaboration (CKD-EPI) equation [25, 26].

Smoking status was categorized as never smoker (never smoked at least 100 cigarettes in their lifetime), former smoker (smoked at least 100 cigarettes in their lifetime but not smoking currently), and current smoker (smokes cigarettes some days or every day). Alcohol intake was based on the self-reported average number of alcoholic drinks in a lifetime and the past year. The equivalent for one alcohol drink used in NHANES was a 12-oz beer, a 5-oz glass of wine, or oneand-half ounces of liquor. Based on the 2015-2020 US dietary guidelines, alcohol intake was categorized as none (never had at least 12 alcoholic drinks in a lifetime or any alcohol in the past year), moderate (one drink for women and not more than two drinks for men in a day), and heavy (more than one or two drinks in a day for women and men, respectively) [27].

Physical activity levels were assessed by calculating the metabolic equivalent of task (MET) minutes per week from the self-reported minutes of moderate-tovigorous intensity physical activity related to leisuretime, work, and transportation (commuting by walking or bicycling). MET scores are used to estimate an activity's intensity, and one MET is defined as the energy expenditure at rest [28]. The MET scores proposed by NHANES for moderate-intensity, vigorous-intensity, and transportation-related physical activity are four, eight, and four, respectively [29]. MET-minutes per week were computed by multiplying the minutes per week of each of the three categories of physical activity (moderate-intensity, vigorous-intensity, and transportation-related physical activity) with their respective MET scores. The MET minutes for the three activities were then summed to compute the total MET minutes per week. The US guidelines on physical activity recommend that adults get at least 75 to $150 \mathrm{~min}$ of vigorous-intensity or 150 to $300 \mathrm{~min}$ of moderate-intensity physical activity weekly [30]. The physical activity level was categorized as none, low, sufficient, and high to correspond to zero, $<600,600-1200$, and $>1200$ METminutes a week, respectively [28].

The "social-level" covariates were all measured at the individual level of analysis and included race/ethnicity (non-Hispanic White, non-Hispanic Black, Hispanic, non-Hispanic Asian, Other), nativity status (US-born, not US-born), marital status (married or living with a partner, unmarried [i.e., never married, separated, divorced, or widowed]), education level (less than high school, high school graduate, some college, college graduate), health insurance (insured, uninsured), employment status, and family income to poverty ratio. Employment status was assessed based on responses to the following survey items: (1) the type of work done the previous week; (2) the number of hours worked in all jobs or businesses the previous week; (3) whether one usually worked $35 \mathrm{~h}$ per week; and (4) the main reason one did not work the previous week. The categories of employment status were: works $<35 \mathrm{~h} /$ week, works $35-44 \mathrm{~h} /$ week, works $\geq 45 \mathrm{~h} /$ week, not working because they are retired, not working due to health reasons, and not working due to other reasons [for example, taking care of family, attending school, or laid off from work]). Family income to poverty ratio was categorized as $<1.00,1.00-1.99,2.00-3.99$, and $\geq 4.00$. 


\section{Statistical analysis and missing data}

In the final analytic sample $(n=5660), 20.1 \%$ of the observations had missing data in one or more covariates. The degree of missingness (unweighted number and weighted percentages) was as follows: marital status $(n=39,0.4 \%)$, education level $(n=9,0.1 \%)$, health insurance status $(n=10,0.2 \%)$, cigarette smoking status $(n=6$, $0.1 \%)$, alcohol intake $(n=403,5.7 \%)$, BMI $(n=90,1.3 \%)$, depressive symptoms $(n=357,5.2 \%)$, and income to poverty ratio $(n=689,9.8 \%)$. Imputation of the missing data was completed using multiple imputation by chained equations (MICE) [31].

All variables in the study (covariates and outcome variable) and NHANES design variables (strata, cluster, and survey weights) were included in the imputation model to avoid generating biased estimates in the analysis model [30,32]. The literature on multiple imputation also recommends having in the imputation model other variables associated with missingness of data to make multiple imputations under the assumptions of missing at random assumptions more plausible [30,33]. The auxiliary variables included in the imputation model included the reference person's age and education level, household size, number of rooms in the household, and homeownership. Stata's mi impute chained command was used to implement the multiple imputation. A total of 40 imputed datasets were generated, and Stata's mi estimate command was used to analyze and pool the results [34].

We did a sensitivity analysis to check for differences between the observed (pre-imputation) data and the complete imputed data (post-imputation) by comparing the subgroup proportions across the variables with missing data. There was minimal or no change in the distribution of pre-imputed and post-imputed data across marital status, education level, health insurance status, BMI, smoking status, depressive symptoms, and alcohol intake. Changes were noted in the income to poverty ratio variable. The proportion of those in the $\geq 4.00$ category decreased from 36.6 to $35.6 \%$ after imputation. The proportion of adults in the $<1.00$ group increased from 12.5 to $13.1 \%$, and the $1.00-1.99$ group increased from 21.1 to $21.7 \%$ (Additional file 1: Supplemental Table 1). The results presented in this report are based on analyses of the complete imputed data.

Survey analysis procedures incorporating the NHANES sample's MEC examination weights and strata and cluster variables were used to account for NHANES's sampling procedures and non-response to obtain results representative of the sampled population [17]. Descriptive analysis was conducted on all covariates for the total study sample. In the bivariate analyses, the distribution of all covariates across the categories of habitual sleep duration was assessed using the $\chi^{2}$ test.
Since the outcome variable (habitual sleep duration) categories are ordered, we examined the ordinal logistic regression model for model fit and violation of statistical assumptions. The approximate likelihood-ratio test of proportionality of odds across response categories and parallel regression assumption test statistics were significant indicating a violation of proportionality of odds assumption. Consequently, a multinomial logistic regression model was used in the analyses. We fit the model using the complete imputed data with habitual sleep duration as the outcome of interest. The multinomial logistic regression models (unadjusted and adjusted) were fitted with all covariates to examine their associations with short $(<7 \mathrm{~h})$ and long sleep duration $(>9 \mathrm{~h})$ using adequate sleep duration $(7-9 \mathrm{~h})$ as the reference category. The multinomial logistic regression model results were compared with complete case analysis results. All data were analyzed using STATA 1C Version 15 [35]. The level of significance for all analyses was set at a $p$-value $<0.05$.

\section{Results \\ Sample population characteristics}

The average age of the participants was 56.1 years; $52.1 \%$ were male; $64.1 \%$ were non-Hispanic White, $13.3 \%$ nonHispanic Black and 12.9\% Hispanic; 33.2\% had some college education, and $26.9 \%$ were college graduates. The prevalence of self-reported adequate sleep duration was $65.7 \%$, while short sleep duration was $23.6 \%$, and long sleep duration was $10.7 \%$. Over a third (37.2\%) had a history of ever telling a doctor or other health professional that they had trouble sleeping. Most $(89.8 \%)$ had health insurance. Over half (51.3\%) had a BMI of $30 \mathrm{~kg} / \mathrm{m}^{2}$ and above; $48.3 \%$ had high self-reported physical activity levels, while $26.2 \%$ were physically inactive. The prevalence of heavy alcohol intake was $32.0 \%$ (Tables 1 and 2).

In bivariate descriptive comparisons, participants with short sleep duration were younger and more likely to be male, non-Hispanic Black, have no health insurance, and work $\geq 45 \mathrm{~h}$ weekly. Conversely, individuals with long sleep duration were older and more likely to be female, not employed, have a high school or lower level of education, have comorbidity or moderate to severe depressive symptoms, and be non-drinkers and physically inactive (Tables 1 and 2).

\section{Individual-level and social-level factors associated with habitual sleep duration}

The multinomial logistic analysis results shown in Tables 3 were derived from the complete data $(n=5660)$ created using multiple imputations, and all associations are presented relative to adequate sleep duration. In the unadjusted multinomial logistic regression results, all 
Table 1 Individual-level characteristics by sleep duration from NHANES (2015-2018) sample of US adults with hypertension ${ }^{\text {a }}$

\begin{tabular}{|c|c|c|c|c|c|}
\hline \multirow[t]{2}{*}{ Characteristics } & \multirow[b]{2}{*}{ Overall } & \multicolumn{3}{|c|}{ Sleep duration } & \multirow[b]{2}{*}{$P$-value } \\
\hline & & Short & Adequate & Long & \\
\hline Total, n (\%) & $5660(100 \%)$ & $1444(23.6)$ & $3495(65.7)$ & $721(10.7)$ & \\
\hline \multicolumn{6}{|l|}{ Sex, \% } \\
\hline Male, & $2927(52.1)$ & 60.0 & 51.3 & 39.7 & \multirow[t]{2}{*}{$<0.001$} \\
\hline Female & $2733(47.9)$ & 40.0 & 48.7 & 60.3 & \\
\hline Age, mean years (SE) & $56.1(0.4)$ & $53.7(0.5)$ & $56.2(0.5)$ & $60.4(0.8)$ & \\
\hline \multicolumn{6}{|l|}{ Age category, $\%$} \\
\hline $18-44$ & $1159(23.8)$ & 26.3 & 23.6 & 18.9 & \multirow[t]{3}{*}{$<0.001$} \\
\hline $45-64$ & $2356(43.7)$ & 51.8 & 42.5 & 33.3 & \\
\hline 65 and above & $2145(32.5)$ & 21.9 & 33.9 & 47.8 & \\
\hline \multicolumn{6}{|l|}{ Comorbidities, \% } \\
\hline Heart disease & $763(11.4)$ & 12.3 & 10.4 & 15.5 & 0.073 \\
\hline Stroke & $361(5.1)$ & 4.5 & 4.6 & 9.6 & $<0.001$ \\
\hline COPD or asthma & $847(14.4)$ & 15.3 & 13.1 & 20.1 & 0.006 \\
\hline Arthritis & $2365(41.8)$ & 40.6 & 40.7 & 50.9 & 0.005 \\
\hline Diabetes mellitus & $1547(22.1)$ & 22.8 & 20.6 & 30.1 & 0.015 \\
\hline Chronic kidney disease & $745(11.2)$ & 9.5 & 10.4 & 20.1 & $<0.001$ \\
\hline \multicolumn{6}{|l|}{ Depressive symptoms, $\%$} \\
\hline Minimal or none & $3933(74.7)$ & 71.7 & 77.7 & 62.4 & \multirow[t]{4}{*}{$<0.001$} \\
\hline Mild & $890(16.5)$ & 16.9 & 15.4 & 22.6 & \\
\hline Moderate & $296(5.8)$ & 7.5 & 4.7 & 8.7 & \\
\hline Moderately severe to severe & $184(3.0)$ & 3.9 & 2.2 & 6.3 & \\
\hline Help-seeking for sleeping difficulty, $\%$ & $1859(37.2)$ & 41.0 & 35.5 & 39.8 & 0.066 \\
\hline \multicolumn{6}{|l|}{ BMI category, \% } \\
\hline$<25$ & $1109(18.1)$ & 17.2 & 18.1 & 20.2 & \multirow[t]{5}{*}{0.439} \\
\hline $25-<30$ & $1755(30.5)$ & 29.2 & 31.0 & 30.7 & \\
\hline $30-<35$ & $1349(25.7)$ & 25.0 & 25.9 & 26.6 & \\
\hline $35-<40$ & $737(13.8)$ & 14.5 & 14.1 & 10.9 & \\
\hline$\geq 40$ & $620(11.8)$ & 14.1 & 10.9 & 11.7 & \\
\hline \multicolumn{6}{|l|}{ Smoking status, $\%$} \\
\hline Never smoker & $3066(52.8)$ & 50.6 & 54.2 & 48.9 & \multirow[t]{3}{*}{$<0.001$} \\
\hline Current smoker & $1005(17.2)$ & 22.1 & 14.7 & 21.3 & \\
\hline Former smoker & $1583(30.0)$ & 27.3 & 31.1 & 29.8 & \\
\hline \multicolumn{6}{|l|}{ Alcohol intake, \% } \\
\hline None & $1973(29.4)$ & 29.8 & 27.6 & 39.2 & \multirow[t]{3}{*}{0.001} \\
\hline Moderate & 1827 (38.6) & 35.8 & 40.4 & 34.4 & \\
\hline Heavy & $1457(32.0)$ & 34.4 & 32.0 & 26.4 & \\
\hline \multicolumn{6}{|l|}{ Physical activity level, \% } \\
\hline Sufficient & $642(11.7)$ & 10.3 & 12.4 & 10.6 & \multirow[t]{4}{*}{$<0.001$} \\
\hline None & $1770(26.2)$ & 26.0 & 24.3 & 38.6 & \\
\hline Low & $803(13.7)$ & 10.6 & 15.0 & 12.8 & \\
\hline High & $2445(48.3)$ & 53.1 & 48.3 & 38.0 & \\
\hline
\end{tabular}

Abbreviations: SE standard error, COPD chronic obstructive pulmonary disease, $B M /$ body mass index

${ }^{a}$ Data are expressed as an unweighted number of participants and weighted percentages and means

covariates except BMI and nativity status showed significant associations with habitual sleep duration (Table 3).

In the fully adjusted multinomial logistic model, age, sex, history of help-seeking for sleeping difficulty, depressive symptoms, and CKD were associated with habitual sleep duration. Older adults ( $\geq 65$ years old) were less likely than young adults (18-44 years) to have short sleep duration (RRR, 0.63; 95\% CI, 0.45-0.91). 
Table 2 Social-level characteristics by sleep duration from NHANES (2015-2018) sample of US adults with hypertension ${ }^{a}$

\begin{tabular}{|c|c|c|c|c|c|}
\hline \multirow[t]{2}{*}{ Characteristics } & \multirow[b]{2}{*}{ Overall } & \multicolumn{3}{|c|}{ Sleep duration } & \multirow[b]{2}{*}{$P$-value } \\
\hline & & Short & Adequate & Long & \\
\hline Total, n (\%) & $5660(100 \%)$ & $1444(23.6)$ & $3495(65.7)$ & $721(10.7)$ & \\
\hline \multicolumn{6}{|l|}{ Race/Ethnicity, \% } \\
\hline Non-Hispanic White & $1905(64.1)$ & 56.4 & 66.9 & 63.6 & \multirow[t]{5}{*}{$<0.001$} \\
\hline Non-Hispanic Black & $1474(13.3)$ & 19.4 & 10.9 & 14.4 & \\
\hline Hispanic & $1381(12.9)$ & 13.9 & 12.4 & 13.8 & \\
\hline Non-Hispanic Asian & $652(5.4)$ & 5.3 & 5.6 & 4.7 & \\
\hline Other & $248(4.3)$ & 5.0 & 4.2 & 3.5 & \\
\hline \multicolumn{6}{|l|}{ Marital status, $\%$} \\
\hline Married/Living with partner & $3301(63.1)$ & 61.7 & 65.1 & 54.3 & \multirow[t]{2}{*}{0.005} \\
\hline Unmarried & $2320(36.9)$ & 38.3 & 34.9 & 45.7 & \\
\hline \multicolumn{6}{|l|}{ Nativity status, $\%$} \\
\hline US-born & $3964(83.2)$ & 81.6 & 83.3 & 85.4 & \multirow[t]{2}{*}{0.211} \\
\hline Not US-born & $1696(16.8)$ & 18.4 & 16.7 & 14.6 & \\
\hline \multicolumn{6}{|l|}{ Education Level, \% } \\
\hline College graduate & $1215(26.9)$ & 23.4 & 29.6 & 18.3 & \multirow[t]{4}{*}{$<0.001$} \\
\hline Some college & $1732(33.2)$ & 36.2 & 33.5 & 24.6 & \\
\hline High school graduate & $1354(26.0)$ & 26.1 & 24.7 & 33.5 & \\
\hline Less than high school & $1350(13.9)$ & 14.3 & 12.2 & 23.6 & \\
\hline \multicolumn{6}{|l|}{ Income to poverty ratio, \% } \\
\hline$\geq 4.00$ & $1165(36.6)$ & 32.9 & 40.2 & 22.3 & \multirow[t]{4}{*}{$<0.001$} \\
\hline $2.00-3.99$ & $1373(29.8)$ & 33.7 & 29.0 & 26.5 & \\
\hline $1.00-1.99$ & $1447(21.1)$ & 20.2 & 20.2 & 28.8 & \\
\hline$<1.00$ & $986(12.5)$ & 13.2 & 10.6 & 22.4 & \\
\hline \multicolumn{6}{|l|}{ Employment Status, \% } \\
\hline Works $35-44 \mathrm{~h} /$ week & $1184(23.8)$ & 23.3 & 26.2 & 10.3 & \multirow[t]{6}{*}{$<0.001$} \\
\hline Works $<35 \mathrm{~h} /$ week & $670(12.1)$ & 9.9 & 13.3 & 9.2 & \\
\hline Works $\geq 45 \mathrm{~h} /$ week & $815(18.5)$ & 28.3 & 17.4 & 3.9 & \\
\hline Not working - health reasons & $743(10.3)$ & 11.1 & 8.1 & 22.4 & \\
\hline Not working - retired & $1623(26.5)$ & 20.5 & 26.5 & 39.7 & \\
\hline Not working - other reasons & $625(8.8)$ & 6.9 & 8.5 & 14.5 & \\
\hline \multicolumn{6}{|l|}{ Has health insurance, $\%$} \\
\hline Yes & $4965(89.8)$ & 86.3 & 91.1 & 89.6 & \multirow[t]{2}{*}{0.003} \\
\hline No & $685(10.2)$ & 13.7 & 8.9 & 10.4 & \\
\hline
\end{tabular}

${ }^{a}$ Data are expressed as an unweighted number of participants and weighted percentages

No age differences were noted in odds for being a long sleeper. Compared to men, women were less likely to be short sleepers (RRR, 0.70; 95\% CI, 0.56-0.88) and more likely to be long sleepers (RRR, 1.24; 95\% CI, 1.001-1.54). Those reporting a history of ever telling a doctor or other healthcare professional they had trouble sleeping were more likely to have short sleep duration (RRR, 1.25; 95\% CI, 1.02-1.53).

Long sleep duration was significantly associated with moderate depressive symptoms (RRR, 1.62; 95\% CI, 1.08-2.44) and moderately severe to severe depressive symptoms (RRR, 1.89; 95\% CI, 1.05-3.43) compared to minimal or no depressive symptoms. In addition, those with CKD were more likely to have a long sleep duration than those with no CKD (RRR, 1.48; 95\% CI, 1.14-1.92). Other comorbid conditions (diabetes mellitus, COPD or current asthma, heart disease, stroke, and arthritis) were not independently associated with habitual sleep duration (Table 3). Similarly, cigarette smoking status, alcohol intake, BMI, and total physical activity level were not independently associated with habitual sleep duration. The physical activity and sleep duration relationship findings remained the same when total physical activity level was substituted with physical activity derived from 
Table 3 Multinomial logistic regression - factors associated with habitual sleep duration in US adults with hypertension

\begin{tabular}{|c|c|c|c|c|c|c|c|c|}
\hline & \multicolumn{4}{|c|}{ Short (vs. adequate) sleep duration } & \multicolumn{4}{|c|}{ Long (vs. adequate) sleep duration } \\
\hline & Crude RRR $(95 \% \mathrm{Cl})^{a}$ & $P$ & $\operatorname{RRR}(95 \% \mathrm{CI})^{b}$ & $P$ & Crude RRR $(95 \% \mathrm{Cl})^{a}$ & $P$ & $\operatorname{RRR}(95 \% \mathrm{Cl})^{b}$ & $P$ \\
\hline \multicolumn{9}{|l|}{ Individual-level factors } \\
\hline \multicolumn{9}{|l|}{ Age } \\
\hline $18-44$ & REF & & REF & & REF & & REF & \\
\hline $45-64$ & $1.09(0.86-1.39)$ & 0.45 & $1.20(0.96,1.51)$ & 0.11 & $0.98(0.68-1.41)$ & 0.90 & $0.72(0.46-1.13)$ & 0.15 \\
\hline$\geq 65$ & $0.58(0.47-0.72)$ & $<0.001$ & $0.63(0.45-0.91)$ & 0.01 & $1.76(1.31-2.36)$ & $<0.001$ & $0.74(0.50-1.09)$ & 0.12 \\
\hline Female (vs. Male) & $0.70(0.58-0.85)$ & 0.001 & $0.70(0.56,0.88)$ & 0.003 & $1.60(1.26-2.03)$ & $<0.001$ & $1.24(1.00-1.54)$ & 0.049 \\
\hline \multicolumn{9}{|l|}{ Comorbidities } \\
\hline $\begin{array}{l}\text { Heart disease (vs. no } \\
\text { heart disease) }\end{array}$ & $1.20(0.82-1.76)$ & 0.33 & $1.20(0.80-1.78)$ & 0.36 & $1.57(1.15-2.17)$ & 0.01 & $0.88(0.59-1.30)$ & 0.50 \\
\hline Stroke (vs. no stroke) & $0.97(0.64-1.48)$ & 0.89 & $0.79(0.54-1.16)$ & 0.21 & $2.18(1.56-3.04)$ & $<0.001$ & $1.09(0.78-1.54)$ & 0.60 \\
\hline $\begin{array}{l}\text { COPD or asthma (vs. no } \\
\text { COPD or asthma) }\end{array}$ & $1.19(0.90-1.58)$ & 0.20 & $1.10(0.83-1.47)$ & 0.49 & $1.67(1.23-2.26)$ & 0.002 & $0.98(0.72-1.35)$ & 0.91 \\
\hline $\begin{array}{l}\text { Arthritis (vs. no } \\
\text { arthritis) }\end{array}$ & $0.99(0.83-1.19)$ & 0.96 & $1.06(0.89-1.26)$ & 0.52 & $1.51(1.17-1.95)$ & 0.002 & $0.93(0.71-1.21)$ & 0.60 \\
\hline $\begin{array}{l}\text { Diabetes mellitus (vs. } \\
\text { no diabetes mellitus) }\end{array}$ & $1.14(0.84-1.52)$ & 0.36 & $1.08(0.83-1.40)$ & 0.55 & $1.66(1.22-2.25)$ & 0.002 & $1.37(1.00-1.88)$ & 0.05 \\
\hline CKD (vs. no CKD) & $0.90(0.68-1.21)$ & 0.49 & $1.18(0.86-1.61)$ & 0.31 & $2.18(1.70-2.79)$ & $<0.001$ & $1.48(1.14-1.92)$ & 0.01 \\
\hline \multicolumn{9}{|l|}{ Depressive symptoms } \\
\hline Minimal or none & REF & & REF & & REF & & REF & \\
\hline Mild & $1.21(0.94-1.56)$ & 0.13 & $1.12(0.88-1.44)$ & 0.34 & $1.83(1.32-2.53)$ & 0.001 & $1.35(0.95-1.93)$ & 0.09 \\
\hline Moderate & $1.73(1.08-2.77)$ & 0.03 & $1.61(0.99-2.61)$ & 0.05 & $2.32(1.63-3.30)$ & $<0.001$ & $1.62(1.08-2.44)$ & 0.02 \\
\hline $\begin{array}{l}\text { Moderately severe } \\
\text { to severe }\end{array}$ & $1.91(1.13-3.23)$ & 0.02 & $1.66(0.88-3.14)$ & 0.11 & $3.62(2.07-6.34)$ & $<0.001$ & $1.89(1.05-3.43)$ & 0.04 \\
\hline $\begin{array}{l}\text { Help-seeking for } \\
\text { sleeping difficulty }\end{array}$ & $1.26(1.02-1.56)$ & 0.03 & $1.25(1.02-1.53)$ & 0.03 & $1.20(0.91-1.59)$ & 0.18 & $0.84(0.61-1.16)$ & 0.28 \\
\hline \multicolumn{9}{|l|}{ Body mass index (BMI) } \\
\hline$<25$ & REF & & REF & & REF & & REF & \\
\hline $25-<30$ & $0.99(0.71-1.38)$ & 0.94 & $0.98(0.70-1.37)$ & 0.88 & $0.89(0.65-1.21)$ & 0.44 & $1.00(0.72-1.39)$ & 0.99 \\
\hline $30-<35$ & $1.01(0.70-1.46)$ & 0.95 & $0.93(0.65-1.34)$ & 0.70 & $0.92(0.61-1.39)$ & 0.68 & $1.06(0.69-1.62)$ & 0.78 \\
\hline $35-<40$ & $1.08(0.75-1.55)$ & 0.67 & $1.02(0.70-1.48)$ & 0.93 & $0.71(0.44-1.12)$ & 0.14 & $0.71(0.42-1.22)$ & 0.21 \\
\hline$\geq 40$ & $1.34(0.99-1.83)$ & 0.06 & $1.19(0.85-1.66)$ & 0.30 & $0.96(0.63-1.46)$ & 0.83 & $0.91(0.56-1.45)$ & 0.67 \\
\hline \multicolumn{9}{|l|}{ Alcohol intake } \\
\hline None & REF & & REF & & REF & & REF & \\
\hline Moderate & $0.83(0.66-1.05)$ & 0.11 & $0.84(0.67-1.05)$ & 0.11 & $0.60(0.47-0.78)$ & $<0.001$ & $0.99(0.76-1.30)$ & 0.96 \\
\hline Heavy & $1.00(0.77-1.30)$ & 0.99 & $0.89(0.68-1.17)$ & 0.38 & $0.58(0.43-0.78)$ & 0.001 & $0.91(0.64-1.31)$ & 0.61 \\
\hline \multicolumn{9}{|l|}{ Cigarette smoking } \\
\hline Never smoker & REF & & REF & & REF & & REF & \\
\hline Current smoker & $1.61(1.24-2.08)$ & 0.001 & $1.25(0.93-1.68)$ & 0.14 & $1.60(1.13-2.29)$ & 0.01 & $1.16(0.82-1.63)$ & 0.39 \\
\hline Former smoker & $0.94(0.76-1.16)$ & 0.55 & $0.88(0.72-1.07)$ & 0.19 & $1.06(0.79-1.42)$ & 0.67 & $0.96(0.72-1.29)$ & 0.78 \\
\hline \multicolumn{9}{|l|}{ Physical activity level } \\
\hline Sufficient & REF & & REF & & REF & & REF & \\
\hline None & $1.28(0.96-1.70)$ & 0.09 & $1.18(0.89-1.57)$ & 0.23 & $1.85(1.27-2.70)$ & 0.002 & $1.39(0.90-2.13)$ & 0.13 \\
\hline Low & $0.85(0.60-1.20)$ & 0.33 & $0.82(0.59-1.15)$ & 0.25 & $1.00(0.66-1.50)$ & 0.99 & $0.91(0.59-1.40)$ & 0.65 \\
\hline High & $1.32(0.98-1.77)$ & 0.07 & $1.14(0.85-1.54)$ & 0.37 & $0.92(0.57-1.46)$ & 0.71 & $1.05(0.65-1.71)$ & 0.84 \\
\hline \multicolumn{9}{|l|}{ Social-level factors } \\
\hline \multicolumn{9}{|l|}{ Race/Ethnicity } \\
\hline Non-Hispanic White & REF & & REF & & REF & & REF & \\
\hline Non-Hispanic Black & $2.11(1.70-2.61)$ & $<0.001$ & $2.05(1.59-2.63)$ & $<0.001$ & $1.38(0.99-1.92)$ & 0.05 & $1.13(0.81-1.57)$ & 0.47 \\
\hline Hispanic & $1.33(1.06-1.67)$ & 0.02 & $1.16(0.82-1.64)$ & 0.39 & $1.17(0.89-1.52)$ & 0.24 & $1.08(0.80-1.46)$ & 0.62 \\
\hline Non-Hispanic Asian & $1.13(0.87-1.46)$ & 0.34 & $1.16(0.74-1.81)$ & 0.51 & $0.89(0.65-1.21)$ & 0.44 & $1.12(0.68-1.84)$ & 0.65 \\
\hline
\end{tabular}


Table 3 (continued)

\begin{tabular}{|c|c|c|c|c|c|c|c|c|}
\hline & \multicolumn{4}{|c|}{ Short (vs. adequate) sleep duration } & \multicolumn{4}{|c|}{ Long (vs. adequate) sleep duration } \\
\hline & Crude RRR $(95 \% \mathrm{Cl})^{\text {a }}$ & $P$ & $\operatorname{RRR}(95 \% \mathrm{Cl})^{\mathrm{b}}$ & $P$ & Crude RRR $(95 \% \mathrm{Cl})^{a}$ & $P$ & $\operatorname{RRR}(95 \% \mathrm{Cl})^{\mathrm{b}}$ & $P$ \\
\hline Other & $1.42(0.97-2.09)$ & 0.07 & $1.24(0.79-1.95)$ & 0.34 & $0.89(0.51-1.56)$ & 0.68 & $0.78(0.48-1.28)$ & 0.31 \\
\hline \multicolumn{9}{|l|}{ Marital status } \\
\hline $\begin{array}{l}\text { Married/Living with } \\
\text { partner }\end{array}$ & REF & & REF & & REF & & REF & \\
\hline Unmarried & $1.16(0.95-1.42)$ & 0.15 & $1.14(0.95-1.37)$ & 0.14 & $1.56(1.20-2.04)$ & 0.002 & $1.06(0.78-1.45)$ & 0.71 \\
\hline \multicolumn{9}{|l|}{ Nativity } \\
\hline US-born & REF & & REF & & REF & & REF & \\
\hline Not US-born & $1.12(0.90-1.40)$ & 0.28 & $1.16(0.83-1.63)$ & 0.37 & $0.86(0.65-1.12)$ & 0.24 & $0.71(0.47-1.07)$ & 0.10 \\
\hline \multicolumn{9}{|l|}{ Education Level } \\
\hline College graduate & REF & & REF & & REF & & REF & \\
\hline Some college & $1.36(1.01-1.84)$ & 0.04 & $1.44(0.92-1.68)$ & 0.15 & $1.19(0.69-2.05)$ & 0.34 & $0.85(0.51-1.43)$ & 0.53 \\
\hline ate High school gradu- & $1.33(0.98-1.81)$ & 0.07 & $1.17(0.85-1.60)$ & 0.32 & $2.20(1.24-3.88)$ & 0.01 & $1.42(0.86-2.34)$ & 0.17 \\
\hline Less than high school & $1.47(1.10-1.97)$ & 0.01 & $1.21(0.88-1.67)$ & 0.24 & $3.14(1.76-5.63)$ & $<0.001$ & $1.59(0.88-2.89)$ & 0.12 \\
\hline \multicolumn{9}{|l|}{ Income to poverty ratio } \\
\hline$\geq 4.00$ & REF & & REF & & REF & & REF & \\
\hline $2.00-3.99$ & $1.38(1.07-1.79)$ & 0.02 & $1.21(0.95-1.56)$ & 0.12 & $1.64(0.99-2.73)$ & 0.06 & $1.20(0.74-1.95)$ & 0.45 \\
\hline $1.00-1.99$ & $1.20(0.92-1.55)$ & 0.17 & $0.94(0.69-1.28)$ & 0.70 & $2.53(1.52-4.19)$ & 0.001 & $1.28(0.77-2.14)$ & 0.33 \\
\hline$<1.00$ & $1.47(1.09-1.97)$ & 0.01 & $0.97(0.67-1.39)$ & 0.85 & $3.70(2.11-6.46)$ & $<0.001$ & $1.45(0.82-2.58)$ & 0.19 \\
\hline \multicolumn{9}{|l|}{ Has health insurance } \\
\hline Yes & REF & & REF & & REF & & REF & \\
\hline No & $1.64(1.24-2.17)$ & 0.001 & $1.30(0.97-1.75)$ & 0.08 & $1.19(0.80-1.76)$ & 0.37 & $1.09(0.75-1.59)$ & 0.65 \\
\hline \multicolumn{9}{|l|}{ Employment Status } \\
\hline Works $35-44 \mathrm{~h} /$ week & REF & & REF & & REF & & REF & \\
\hline Works $<35 \mathrm{~h} /$ week & $0.84(0.58-1.20)$ & 0.32 & $0.88(0.61-1.28)$ & 0.50 & $1.77(1.15-2.73)$ & 0.01 & $1.71(1.07-2.73)$ & 0.03 \\
\hline Works $\geq 45 \mathrm{~h} /$ week & $1.83(1.34-2.51)$ & 0.001 & $1.81(1.32-2.48)$ & 0.001 & $0.58(0.28-1.19)$ & 0.13 & $0.66(0.34-1.29)$ & 0.21 \\
\hline $\begin{array}{l}\text { Not working - } \\
\text { Health reasons }\end{array}$ & $1.55(1.10-2.18)$ & 0.02 & $1.12(0.77-1.63)$ & 0.53 & $7.09(4.62-10.9)$ & $<0.001$ & $4.87(2.89-8.22)$ & $<0.001$ \\
\hline $\begin{array}{l}\text { Not working - } \\
\text { Retired }\end{array}$ & $0.87(0.69-1.09)$ & 0.21 & $1.41(1.01-1.98)$ & 0.04 & $3.82(2.42-6.04)$ & $<0.001$ & $3.46(2.18-5.49)$ & $<0.001$ \\
\hline $\begin{array}{l}\text { Not working - Other } \\
\text { reason }\end{array}$ & $0.91(0.70-1.19)$ & 0.48 & $0.85(0.65-1.12)$ & 0.23 & $4.33(2.66-7.05)$ & $<0.001$ & $3.29(1.84-5.88)$ & $<0.001$ \\
\hline
\end{tabular}

Abbreviations: $R R R$ relative risk ratio, $P p$-value, COPD chronic obstructive pulmonary disease

a Unadjusted model

${ }^{\mathrm{b}}$ Adjusted model - each covariate adjusted for all other variables in the table

leisure-time and transport-related activities in the multinomial logistic model.

Non-Hispanic Black adults with hypertension were twice as likely as their non-Hispanic White counterparts to have short sleep duration (RRR, 2.08; 95\% CI, 1.612.67). The odds of being a short or long sleeper in Hispanic, non-Hispanic Asian, and Other race/ethnic groups were not significantly different from that of the non-Hispanic White race/ethnic group (Table 3).

In the association between employment status and habitual sleep duration, individuals working for $\geq 45 \mathrm{~h} /$ week were more likely to have short sleep duration than those working 35-44h/week (RRR, 1.81; 95\% CI,
$1.32-2.48)$. Those retired were more likely to be short sleepers (RRR, 1.41; 95\% CI, 1.01-1.98) or long sleepers (RRR, 3.46; 95\% CI, 2.18-5.49) than those working $35-44 \mathrm{~h} /$ week. Long sleep duration was also more likely in those not working due to health reasons (RRR, 4.87; 95\% CI, 2.89-8.22) and those not working due to other reasons such as attending school and taking care of the family (RRR, 3.29; 95\% CI, 1.84-5.88) compared to those working 35-44h/week. Marital status, education level, and health insurance did not show independent associations with habitual sleep duration.

The adjusted multinomial logistic regression results from the complete imputed data model $(n=5660)$ were 
compared to those of complete case analysis $(n=4520)$. The direction and significance of the relationship between the covariates and habitual sleep duration in the two models were similar to except in employment status, help-seeking for sleeping difficulty, and depressive symptoms covariates where the level of significance differed (Additional file 2: Supplemental Table 2). In employment status, retirement was associated with short sleep in the imputed model (RRR, 1.41; 95\% CI, 1.01-1.98) but not in complete case analysis (RRR, 1.39; 95\% CI, 0.94-2.06). Help-seeking for sleeping difficulty was associated with short sleep in the imputed model (RRR,1.25; 95\% CI, 1.02-1.53) but not in complete case analysis (RRR,1.20; 95\% CI, 0.97-1.50). Finally, moderate depressive symptoms were associated with short sleep duration in the complete case analysis (RRR, 1.87; 95\% CI, 1.23-2.85) but not in the imputed model (RRR, 1.61; 95\% CI, 0.99-2.61).

Results from the complete case analyses showed no significant associations between depressive symptoms and long sleep duration, but in the complete imputed data model, long sleep duration was associated with both moderate (RRR,1.62; 95\% CI, 1.08-2.44 versus RRR, 1.52; 95\% CI, 0.98-2.37 in the complete case analysis) and moderately severe to severe depressive symptoms (RRR,1.89; 95\% CI, $1.05-3.43$ vs. RRR, 1.78; 95\% CI, 0.84-3.76 in the complete case analysis). The results show that the differences noted were on the significance of the relationship between the specified variables and habitual sleep duration but not on the direction of the relationships. The complete case analysis model included 4520 observations (compared to 5660 observations for complete imputed data), translating to a $20 \%$ loss in information. This loss of information may bias results in the complete case analysis and may have contributed to the differences noted between the two models. Some of the differences observed could also be due to an increase in power in the imputed data model.

\section{Discussion}

In this large nationally representative sample of adults with hypertension, we find numerous sociodemographic and health characteristics are significantly associated with habitual sleep duration. Among US adults with hypertension, help-seeking for sleeping difficulty, defined in the present study as a history of telling a doctor or other health professional that one had trouble sleeping, was independently associated with short sleep duration. Previous research has also noted significant associations between self-reported difficulties initiating or maintaining sleep and short sleep duration [36]. These findings have important implications because short sleep duration and other sleep problems, such as insomnia synergistically increase the risk for adverse cardiovascular outcomes [37, 38]. The significant association between help-seeking for sleeping difficulty and short sleep duration among those with hypertension provides a point of intervention for health care teams to comprehensively assess and support all aspects of sleep health, including habitual sleep duration, in patients who report sleep problems.

Findings from studies with other populations showed no differences by gender in habitual sleep duration [13, 14]. In contrast, we found that women with hypertension were less likely to be short sleepers and more likely to be long sleepers than men with hypertension. The inconsistency may be partly due to the different approaches used in measuring sleep duration. The two studies defined sleep duration based on the amount of sleep obtained in a day [14] or $24 \mathrm{~h} \mathrm{[13],} \mathrm{increasing} \mathrm{the} \mathrm{likelihood} \mathrm{that} \mathrm{nap-}$ ping time was captured. In contrast, our study measured the amount of sleep obtained in the main sleep period. Studies have reported a higher frequency of napping among men $[39,40]$. The differences in napping may account for sex differences in sleep duration when the assessment is limited to the amount of sleep obtained during the main sleep period.

The relationship between chronic disease and sleep duration varies across studies. Consistent with other studies [41, 42], we found that CKD was associated with long sleep duration but not short sleep duration. Other previous research findings found that both short $(<6 \mathrm{~h} /$ night) and long sleep duration ( $>8 \mathrm{~h} /$ night) were positively associated with incident CKD with the strongest association occurring in those who habitually slept $<4 \mathrm{~h}$ per night [43]. The increased risk of CKD in short sleepers may be due to the increased sympathetic nervous system activity and inflammation that occurs with sleep loss [44].

On the other hand, there is minimal evidence of a pathway through which long sleep duration can cause disease. In those with chronic disease, the long sleep duration may be due to ill health or the underlying pathophysiological mechanisms of the specific condition, such as increased inflammation $[1,45]$. The inflammatory processes can cause excessive fatigue and sleepiness [45, 46] and contribute to more time spent in bed. Therefore, the inclusion of markers of pathophysiological processes such as inflammatory markers in future studies would provide more insight into the mechanisms underlying the relationship between disease and sleep duration.

Similar to our findings in adults with hypertension, many previous studies in the general population have found no association between habitual sleep duration and COPD, heart disease, diabetes $[16,47]$, stroke $[16,47]$, or arthritis $[13,16]$. However, other studies found that short sleep duration was significantly associated with COPD 
[13], heart disease [13, 14], and arthritis [47], while stroke was associated with long sleep duration [43]. The inconsistencies across studies may be partly related to differences in baseline characteristics of study participants, the varied ways in which sleep duration is measured and analyzed, differences in the proportion of those with comorbidities, and other factors controlled in the studies.

Consistent with prior findings across different adult populations, including older adults and those attending outpatient care settings [16, 36, 48, 49], we found that moderate to severe depressive symptoms were associated with long sleep duration. Other findings have also shown an association between sleeping less than $6 \mathrm{~h}$ and risk for depression [49] which may point to a dose-response relationship between short sleep and depression. Overall, the literature points to a bidirectional relationship between sleep duration and mental health indicators, including depressive symptoms. Circadian rhythm disruptions and insomnia are common in depression and can contribute to short sleep [50]. Sleep loss has also been linked to impairment in emotional regulation and decreased positive and increased negative emotions, which can trigger and worsen depressive symptoms [51, 52]. Depression has also been linked to elevated levels of pro-inflammatory markers [53, 54], whose effects include excessive fatigue and sleepiness $[46,54,55]$. Hypertension is also associated with increased inflammation $[56,57]$. Therefore, having both hypertension and depression may worsen the effects of inflammation in the body.

The relationship between sleep duration and other health-related behaviors has been examined in many studies. Consistent with prior studies, we found no significant relationship between alcohol intake and habitual sleep duration $[13,16]$. Other previous findings have shown heavy alcohol intake to be associated with sleep continuity disturbances but not sleep duration [58]. Alcohol intake near bedtime can affect sleep quality because it disrupts the normal sleep architecture [59]. Consistent with previous studies, we found no association between cigarette smoking and habitual sleep duration $[13,16]$.

Research findings on the relationship between physical activity and sleep duration are mixed. Similar to a prior study in older adults [13], we noted no associations between physical activity and sleep duration in adults with hypertension. Other findings suggest that being physically active is associated with reduced odds of having a long sleep duration [14, 60]. The negative association between physical activity and long sleep duration may be related to other confounding factors, such as health conditions, not controlled for in studies. Findings from experimental studies suggest that physical activity substantially improves other sleep parameters such as sleep regularity and sleep quality but only has modest effects on total sleep time [61,62]. On the other hand, adequate sleep can enhance the ability to remain physically active because it improves mental and physical performance [63].

We noted that individuals working $\geq 45 \mathrm{~h}$ per week were more likely to be short sleepers, while those working $<35 \mathrm{~h}$ per week or not working were more likely to be long sleepers when compared to individuals working 35-44h per week. Previous studies also have found significant associations between longer work hours and short sleep duration [64, 65]. Findings from the American Time Use Survey showed that the main activity exchanged for sleep is paid work [66], and other results show that a reduction in work hours leads to a significant increase in sleep duration [65]. In the present study, those in retirement also had a slight but significant increase in odds of being a short sleeper than those working 35-44h/ week. These findings on retirement and sleep duration may be partly explained by how sleep duration was quantified in our study. The present study only considered sleep obtained during the main sleep period. Studies have found that individuals who have transitioned from work to retirement tend to nap more frequently or for longer durations when compared to those who are employed $[67,68]$.

Our results on the relationship between race/ethnicity and habitual sleep duration among adults with hypertension are partly consistent with previous findings in the general US adult population. Consistent with other studies [69-71], we found that non-Hispanic Black adults were more likely than non-Hispanic White adults to be short sleepers. However, prior studies also reported significantly higher odds for short sleep duration among Hispanic [71] and non-Hispanic Asian adults than non-Hispanic White adults [70, 71]. In contrast, our study showed that the odds of being a short or long sleeper in Hispanic or non-Hispanic Asian adults were not significantly different from that of nonHispanic White adults. Of note, in these studies, the strongest association between race/ethnicity and short sleep duration has been observed in non-Hispanic Black adults [70, 71].

Previous studies have also found objectively measured total sleep time of non-Hispanic Black US adults to be significantly shorter than that of non-Hispanic White, Hispanic, and non-Hispanic Asian US adults [69, 71]. These findings on non-Hispanic Black race/ethnicity and short sleep duration are noteworthy because hypertension is more prevalent among non-Hispanic Black adults than in non-Hispanic White, Hispanic, or nonHispanic Asian adults [20]. Previous findings suggest that the racial/ethnic differences in sleep duration may be related to differences in stress and perceived racial 
discrimination [15, 72]. Further research is needed to explore further the mechanisms underlying these racial/ ethnic differences in sleep duration.

Despite this study being nationally representative, there are limitations to be noted. The study was cross-sectional, and consequently, we cannot infer any temporality of the relationships between predictors such as chronic health conditions and habitual sleep duration. Second, NHANES did not have data on other potential predictors of sleep duration, such as inflammatory biomarkers, stress, sleep attitudes, napping, and caregiving $[15,16,39$, $73,74]$. Third, data for many variables used in this study, including habitual sleep duration, chronic health conditions, and health behaviors, were measured using selfreports. Even though the collection of self-reported data is more feasible than objective measurements in largescale surveys such as NHANES, it increases the risk for measurement error related to factors such as poor recall and social desirability [75]. To reduce measurement error, part of the NHANES protocols involved clarifying with participants any extreme high or low self-reports on various questionnaire items. For example, for sleep duration, NHANES interviewers verified with participants sleep duration $\leq 4$ or $\geq 10 \mathrm{~h}$.

Despite the limitations noted, the information gathered on factors associated with short and long sleep duration in adults with hypertension provides important contributions to the literature on predictors of poor sleep outcomes in this population. Future studies in hypertensive adults should consider using objective measures of sleep duration and other health behaviors, such as physical activity, and include other important predictors of sleep duration in their analyses.

\section{Conclusion}

This study used data from a national survey to assess the contribution of sociodemographic and health factors related to habitual sleep duration in adults with hypertension. In adults with hypertension, the factors positively associated with short sleep duration included helpseeking for sleeping difficulty, non-Hispanic Black race/ ethnicity, working 45 or more hours per week, and being in retirement. These factors provide information about hypertensive adults at most risk for poor health outcomes related to inadequate sleep's adverse effects. Such evidence can help develop tailored approaches for supporting healthy sleep habits, including adequate habitual duration, in adults with hypertension. In addition, future intervention studies aimed at promoting healthy sleep habits in groups with high rates of short sleep duration will help identify how sleep impacts health outcomes. Also, intervention studies in adults with hypertension are needed to identify modifiable factors that improve habitual sleep duration.

\section{Abbreviations}

AASM/SRS: American Academy of Sleep Medicine and Sleep Research Society; ACC/AHA: American College of Cardiology and American Heart Association; BP: Blood pressure; BMI: Body mass index; CDC: Centers for Disease Control and Prevention; COPD: Chronic obstructive pulmonary disease; $\mathrm{Cl}$ : Confidence interval; CKD: Chronic kidney disease; eGFR: Estimated glomerular filtration rate; CKD-EPI: Chronic Kidney Disease Epidemiology Collaboration; MET: Metabolic equivalent of task; MICE: Multiple imputation by chained equations; MEC: Mobile examination center; NHANES: National Health and Nutrition Examination Survey; NSF: National Sleep Foundation; PHQ-9: 9-item Patient Health Questionnaire; RRR: Relative risk ratio; US: United States; WHO: World Health Organization.

\section{Supplementary Information}

The online version contains supplementary material available at https://doi. org/10.1186/s12889-021-12465-2.

Additional file 1: Supplemental Table 1. Comparative sensitivity analysis between pre-imputation and post-imputation data of variables with missing data.

Additional file 2: Supplemental Table 2. Comparison of results on factors associated with short and long sleep duration - complete case analysis and post-imputation.

\section{Acknowledgments}

Not applicable.

\section{Authors' contributions}

E.G.O. conceptualized and designed the study, downloaded the NHANES data, analyzed the data, and drafted the manuscript. S.L.C., J.F.B., C.D., J.T.B., and J.E.G. supervised the conceptualization and design of the study, the analysis of data, and reviewed and edited the manuscript. All authors read and approved the

final manuscript.

\section{Funding}

This project was supported by Betty Irene Moore School of Nursing grant number 4294.

\section{Availability of data and materials}

The datasets generated and/or analyzed during the current study are available in the Centers for Disease Control and Prevention (CDC) NHANES repository, https://wwwn.cdc.gov/nchs/nhanes/.

\section{Declarations}

\section{Ethics approval and consent to participate}

The National Center for Health Statistics Ethics Review Board approved the NHANES protocol, and informed consent was obtained from the participants. The current study was deemed exempt from further review because the data used is de-identified and publicly accessible.

\section{Consent for publication}

Not applicable.

\section{Competing interests}

The authors declare that they have no competing interests.

\section{Author details}

${ }^{1}$ Betty Irene Moore School of Nursing, University of California, Davis 2570 48th Street, Sacramento, CA 95817, USA. ²Department of Statistics, University of California, Davis 399 Crocker Ln, Davis, CA 95616, USA. ${ }^{3}$ Department of Psychiatry, Columbia University, 1051 Riverside Drive, New York, NY 10032, USA. 
Received: 22 June 2021 Accepted: 20 December 2021

Published online: 06 January 2022

\section{References}

1. Watson NF, Badr MS, Belenky G, Bliwise DL, Buxton OM, Buysse D, et al. Joint consensus statement of the American Academy of sleep medicine and Sleep Research Society on the recommended amount of sleep for healthy adult: methodology and discussion. Sleep. 2015;38(8):1161-83.

2. Hirshkowitz M, Whiton $K$, Albert SM, Alessi C, Bruni O, DonCarlos L, et al. National Sleep Foundation's updated sleep duration recommendations: final report. Sleep Health. 2015;1 (4):233-43.

3. Khubchandani J, Price JH. Short sleep duration in working American adults, 2010-2018. J Community Health. 2020;45(2):219-27.

4. Centers for Disease Control and Prevention. Hypertension Cascade: hypertension prevalence, treatment and control estimates among US adults aged 18 years and older applying the criteria from the American College of Cardiology and American Heart Association's 2017 hypertension quideline-NHANES 2015-2018. Atlanta: U.S. Department of Health and Human Services; 2021.

5. Whelton PK, Carey RM, Aronow WS, Casey DE Jr, Collins KJ, Dennison Himmelfarb C, et al. 2017 ACC/AHA/AAPA/ABC/ACPM/AGS/APhA/ASH/ ASPC/NMA/PCNA guideline for the prevention, detection, evaluation, and Management of High Blood Pressure in adults: a report of the American College of Cardiology/American Heart Association task force on clinical practice guidelines. Hypertension. 2018;71(6):e13-e115.

6. Li H, Ren Y, Wu Y, Zhao X. Correlation between sleep duration and hypertension: a dose-response meta-analysis. J Hum Hypertens. 2019;33(3):218-28.

7. Grandner M, Mullington JM, Hashmi SD, Redeker NS, Watson NF, Morgenthaler TI. Sleep duration and hypertension: analysis of $>700,000$ adults by age and sex. J Clin Sleep Med. 2018;14(6):1031-9.

8. Cabeza de Baca T, Chayama KL, Redline S, Slopen N, Matsushita F, Prather AA, et al. Sleep debt: the impact of weekday sleep deprivation on cardiovascular health in older women. Sleep. 2019;42(10):zsz149.

9. Matthews EE, Li C, Long CR, Narcisse MR, Martin BC, McElfish PA. Sleep deficiency among native Hawaiian/Pacific islander, black, and White Americans and the association with cardiometabolic diseases: analysis of the National Health Interview Survey Data. Sleep Health. 2018;4(3):273-83.

10. Cassidy S, Chau JY, Catt M, Bauman A, Trenell MI. Low physical activity, high television viewing and poor sleep duration cluster in overweight and obese adults; a cross-sectional study of 398,984 participants from the UK biobank. Int J Behav Nutr Phys Act. 2017;14(1):57.

11. Loredo JS, Weng J, Ramos AR, Sotres-Alvarez D, Simonelli G, Talavera GA et al. Sleep patterns and obesity: Hispanic community health study/study of Latinos Sueno Ancillar study. Chest. 2019;156(2):348-56.

12. Mondin TC, Stuart AL, Williams LJ, Jacka FN, Pasco JA, Ruusunen A. Diet quality, dietary patterns and short sleep duration: a cross-sectional population-based study. Eur J Nutr. 2019:58(2):641-51.

13. Wang S, Wu Y, Ungvari GS, Ng CH, Forester BP, Gatchel JR, et al. Sleep duration and its association with demographics, lifestyle factors, poor mental health and chronic diseases in older Chinese adults. Psychiatry Res. 2017:257:212-8.

14. Ren Y, Liu Y, Meng T, Liu W, Qiao Y, Gu Y, et al. Social-biological influences on sleep duration among adult residents of northeastern China. Health Qual Life Outcomes. 2019;17(1):47.

15. Alcantara C, Patel SR, Carnethon M, Castaneda S, Isasi CR, Davis $S$, et al. Stress and sleep: results from the Hispanic community health study/study of Latinos sociocultural ancillary study. SSM Popul Health. 2017;3:713-21.

16. Smagula SF, Harrison S, Cauley JA, Ancoli-Israel S, Cawthon PM, Cummings $S$, et al. Determinants of change in objectively assessed sleep duration among older men. Am J Epidemiol. 2017:185(10):933-40.

17. Zipf G, Chiappa M, Porter KS, Ostchega Y, Lewis BG, Dostal J. National Health and Nutrition Examination Survey: Plan and operations, 19992010. In: Vital Health and Statistics. vol. 1(56): National Center for Health Statistics: 2013

18. Centers for Disease Control and Prevention. National Center for Health Statistics. NHANES Response Rates and Population Totals. Hyattsville: U.S Department of Health and Human Services, Centers for Disease Control and Prevention; [https://wwwn.cdc.gov/nchs/nhanes/responserates. aspx]. Accessed 22 July 2019.

19. Centers for Disease Control and Prevention. National Center for Health Statistics. National Health and Nutrition Examination Survey: Physician Examination Procedures Manual. Hyattsville: U.S. Department of Health and Human Services, Centers for Disease Control and Prevention; 2018] [https://wwwn.cdc.gov/nchs/data/nhanes/2017-2018/manuals/2018Physician-Examination-Manual-508.pdf].

20. Dorans KS, Mills KT, Liu Y, He J. Trends in prevalence and control of hypertension according to the 2017 American College of Cardiology/ American Heart Association (ACC/AHA) guideline. J Am Heart Assoc. 2018;7(11):e008888.

21. Ostchega Y, Zhang G, Hughes JP, Nwankwo T. Factors associated with hypertension control in US adults using 2017 ACC/AHA guidelines: National Health and nutrition examination survey 1999-2016. Am J Hypertens. 2018;31(8):886-94.

22. Grandner MA. Social-ecological model of sleep health. In: Grandner MA editor. Sleep and health. 1st ed: Elsevier Inc; 2019. p. 45-53.

23. World Health Organization: Body mass index - BMI. https://www.euro. who.int/en/health-topics/disease-prevention/nutrition/a-healthy-lifes tyle/body-mass-index-bmi. Accessed 18 Feb 2020.

24. Kroenke K, Spitzer RL, Williams JBW. The PHQ-9: validity of a brief depression severity measure. J Gen Intern Med. 2001;16:606-13.

25. Levey AS, Stevens LA, Schmid CH, Zhang YL, Castro AF, Feldman HI, et al. A new equation to estimate glomerular filtration rate. Ann Intern Med. 2009;150(9):604-12.

26. Murphy D, McCulloch CE, Lin F, Banerjee T, Bragg-Gresham JL, Eberhardt MS, et al. Trends in prevalence of chronic kidney disease in the United States. Ann Intern Med. 2016;165(7):473-81.

27. U.S. Department of Health and Human Services, U.S. Department of Agriculture: 2015-2020 Dietary Guidelines for Americans. 8th ed; 2015.

28. 2018 Physical Activity Guidelines Advisory Committee. 2018 Physical Activity Guidelines Advisory Committee Scientific Report. Washington, DC: U.S. Department of Health and Human Services; 2018.

29. Centers for Disease Control and Prevention, National Center for Health Statistics: National Health and nutrition examination survey 2017-2018 data documentation, codebook, and frequencies: physical activity (PAQ J); 2020.

30. White IR, Royston P, Wood AM. Multiple imputation using chained equations: issues and guidance for practice. Stat Med. 2011;30(4):377-99.

31. Royston P, White IR. Multiple imputation by chained equations (MICE): implementation in Stata. J Stat Softw. 2011:45(4):1-20.

32. Quartagno M, Carpenter JR, Goldstein H. Multiple imputation with survey weights: a multilevel approach. J Surv Stat Methodol. 2020;8(5):965-89.

33. Nguyen $C D$, Carlin JB, Lee KJ. Model checking in multiple imputation: an overview and case study. Emerg Themes Epidemiol. 2017;14(1):8.

34. StataCorp. Stata Multiple-Imputation Reference Manual. Release 15. College Station: StataCorp LLC; 2017.

35. StataCorp. Stata User's guide release 15. College Station: StataCorp LLC. 2017.

36. Zheng W, Luo XN, Li HY, Ke XY, Dai Q, Zhang CJ, et al. Association of sleep duration with sleep disturbances, quality of life, and sociodemographic factors in general hospital outpatients. Perspect Psychiatr Care. 2018;54(3):422-7.

37. Bertisch SM, Pollock BD, Mittleman MA, Buysse DJ, Bazzano LA, Gottlieb DJ, et al. Insomnia with objective short sleep duration and risk of incident cardiovascular disease and all-cause mortality: sleep heart health study. Sleep. 2018;41(6):1-9.

38. Pepin J-L, Borel A-L, Tamisier R, Baguet J-P, Levy P, Dauvilliers Y. Hypertension and sleep: overview of a tight relationship. Sleep Med Rev. 2014;18(6):509-19.

39. Furihata R, Kaneita Y, Jike M, Ohida T, Uchiyama M. Napping and associated factors: a Japanese nationwide general population survey. Sleep Med. 2016:20:72-9.

40. Leng Y, Wainwright NWJ, Cappuccio FP, Surtees PG, Hayat S, Luben R, et al. Daytime napping and increased risk of incident respiratory diseases: symptom, marker, or risk factor? Sleep Med. 2016;23:12-5.

41. Kim C-W, Chang Y, Sung E, Yun KE, Jung H-S, Ko B-J, et al. Sleep duration and quality in relation to chronic kidney disease and glomerular hyperfiltration in healthy men and women. PLoS One. 2017;12(4):e0175298. 
42. Choi H, Kim HC, Lee JY, Lee J-M, Choi DP, Suh I. Sleep duration and chronic kidney disease: the Korean genome and epidemiology study (KoGES)-Kangwha study. Korean J Intern Med. 2017;32(2):323-34.

43. Kim M-Y, Lee S, Myong YH, Lee YJ, Kim M-R, Shin J-S, et al. Association between sleep duration and stroke prevalence in Korean adults: a cross sectional study. BMJ Open. 2018;8(6):e021491.

44. Bo Y, Yeoh E-k, Guo C, Zhang Z, Tam T, Chan T-C, et al. Sleep and the risk of chronic kidney disease: a cohort study. J Clin Sleep Med. 2019:15(03):393-400.

45. Gangwisch JE. A review of evidence for the link between sleep duration and hypertension. Am J Hypertens. 2014;27(10):1235-42.

46. Krueger JM. The role of cytokines in sleep regulation. Curr Pharm Des. 2008;14(32):3408-16.

47. Chang VC, Chaput JP, Roberts KC, Jayaraman G, Do MT. Factors associated with sleep duration across life stages: results from the Canadian health measures survey. Health Promot Chronic Dis Prev Can. 2018;38(11):404-18.

48. Basnet S, Merikanto I, Lahti T, Mannisto S, Laatikainen T, Vartiainen E, et al. Associations of common chronic non-communicable diseases and medical conditions with sleep-related problems in a population-based health examination study. Sleep Sci. 2016;9(3):249-54.

49. Mohan J, Xiaofan G, Yingxian S. Association between sleep time and depression: a cross-sectional study from countries in rural northeastern China. J Int Med Res. 2017:45(3):984-92.

50. Sun Y, Shi L, Bao Y, Sun Y, Shi J, Lu L. The bidirectional relationship between sleep duration and depression in community-dwelling middleaged and elderly individuals: evidence from a longitudinal study. Sleep Med. 2018;52:221-9.

51. Palmer CA, Alfano CA. Sleep and emotion regulation: an organizing, integrative review. Sleep Med Rev. 2017;31:6-16.

52. Watling J, Pawlik B, Scott K, Booth S, Short MA. Sleep loss and affective functioning: more than just mood. Behav Sleep Med. 2017;15(5):394-409.

53. Osimo EF, Pillinger T, Rodriguez IM, Khandaker GM, Pariante CM, Howes OD. Inflammatory markers in depression: a meta-analysis of mean differences and variability in 5,166 patients and 5,083 controls. Brain Behav Immun. 2020;87:901-9.

54. Pedraz-Petrozzi B, Neumann E, Sammer G. Pro-inflammatory markers and fatigue in patients with depression: a case-control study. Sci Rep. 2020;10(1):9494

55. Rohleder N, Aringer M, Boentert M. Role of interleukin- 6 in stress, sleep, and fatigue. Ann NY Acad Sci. 2012;1261(1):88-96.

56. De Miguel C, Rudemiller NP, Abais JM, Mattson DL. Inflammation and hypertension: new understandings and potential therapeutic targets. Curr Hypertens Rep. 2015;17(1):507.

57. Bautista LE, Vera LM, Arenas IA, Gamarra G. Independent association between inflammatory markers (C-reactive protein, interleukin-6, and TNF-a) and essential hypertension. J Hum Hypertens. 2005;19(2):149-54

58. Britton A, Fat LN, Neligan A. The association between alcohol consumption and sleep disorders among older people in the general population. Sci Rep. 2020;10(1):5275

59. Chakravorty S, Chaudhary NS, Brower KJ. Alcohol dependence and its relationship with insomnia and other sleep disorders. Alcohol Clin Exp Res. 2016:40(11):2271-82.

60. Smagula SF, Koh WP, Wang R, Yuan JM. Chronic disease and lifestyle factors associated with change in sleep duration among older adults in the Singapore Chinese health study. J Sleep Res. 2016;25(1):57-61.

61. Quist JS, Rosenkilde M, Gram AS, Blond MB, Holm-Petersen D, Hjorth MF, et al. Effects of exercise domain and intensity on sleep in women and men with overweight and obesity. J Obes. 2019;2019:2189034.

62. Kredlow MA, Capozzoli MC, Hearon BA, Calkins AW, Otto MW. The effects of physical activity on sleep: a meta-analytic review. J Behav Med. 2015;38(3):427-49.

63. Chennaoui M, Arnal PJ, Sauvet F, Leger D. Sleep and exercise: a reciprocal issue? Sleep Med Rev. 2015;20:59-72.

64. Berkman LF, Liu SY, Hammer L, Moen P, Klein LC, Kelly E, et al. Work-family conflict, cardiometabolic risk, and sleep duration in nursing employees. J Occup Health Psychol. 2015;20(4):420-33.

65. Barger LK, Sullivan JP, Blackwell T, O'Brien CS, St. Hilaire MA, Rahman SA, et al. Effects on resident work hours, sleep duration, and work experience in a randomized order safety trial evaluating resident-physician schedules (ROSTERS). Sleep. 2019;42(8):zsz110.
66. Basner M, Spaeth AM, Dinges DF. Sociodemographic characteristics and waking activities and their role in the timing and duration of sleep. Sleep. 2014:37(12):1889-906.

67. Harden CM, Peppard PE, Palta M, Barnet JH, Hale L, Nieto FJ, et al. Oneyear changes in self-reported napping behaviors across the retirement transition. Sleep Health. 2019;5(6):639-46.

68. Jing $\mathrm{R}$, Barath $\mathrm{D}, \mathrm{Zhang} \mathrm{H}$, Chen J, Fang $\mathrm{H}$. Changes in sleep duration associated with retirement transitions: the role of naps. J Sleep Res. 2020:29(6):e12975.

69. Carnethon MR, De Chavez PJ, Zee PC, Kim KY, Liu K, Goldberger JJ, et al. Disparities in sleep characteristics by race/ethnicity in a population-based sample: Chicago area sleep study. Sleep Med. 2016;18:50-5.

70. Cunningham TJ, Wheaton AG, Ford ES, Croft JB. Racial/ethnic disparities in self-reported short sleep duration among US-born and foreign-born adults. Ethn Health. 2016;21(6):628-38.

71. Chen X, Wang R, Zee P, Lutsey PL, Javaheri S, Alcantara C, et al. Racial/ ethnic differences in sleep disturbances: the multi-ethnic study of atherosclerosis (MESA). Sleep. 2015;38(6):877-88.

72. Slopen N, Lewis TT, Williams DR. Discrimination and sleep: a systematic review. Sleep Med. 2016:18:88-95.

73. Liu Y, Wheaton AG, Edwards VJ, Xu F, Greenlund KJ, Croft JB. Short selfreported sleep duration among caregivers and non-caregivers in 2016. Sleep Health. 2020;6(5):651-6.

74. Ruggiero AR, Peach HD, Gaultney JF. Association of sleep attitudes with sleep hygiene, duration, and quality: a survey exploration of the moderating effect of age, gender, race, and perceived socioeconomic status. Health Psychol Behav Med. 2019:7(1):19-44.

75. Althubaiti A. Information bias in health research: definition, pitfalls, and adjustment methods. J Multidiscip Healthc. 2016;9:211-7.

\section{Publisher's Note}

Springer Nature remains neutral with regard to jurisdictional claims in published maps and institutional affiliations.

Ready to submit your research? Choose BMC and benefit from:

- fast, convenient online submission

- thorough peer review by experienced researchers in your field

- rapid publication on acceptance

- support for research data, including large and complex data types

- gold Open Access which fosters wider collaboration and increased citations

- maximum visibility for your research: over 100M website views per year

At BMC, research is always in progress.

Learn more biomedcentral.com/submissions 